ARTICLE

Received 2 Jun 2014 | Accepted 10 Oct 2014 | Published 24 Nov 2014

\title{
Experimental demonstration of spinor slow light
}

\author{
Meng-Jung Lee ${ }^{1}$, Julius Ruseckas², Chin-Yuan Lee ${ }^{1}$, Viačeslav Kudriašov², Kao-Fang Chang1, \\ Hung-Wen $\mathrm{Cho}^{1}$, Gediminas Juzeliūnas ${ }^{2} \&$ Ite $\mathrm{A}$. $\mathrm{Yu}^{1}$
}

Slow light based on the effect of electromagnetically induced transparency is of great interest due to its applications in low-light-level nonlinear optics and quantum information manipulation. The previous experiments all dealt with the single-component slow light. Here, we report the experimental demonstration of two-component or spinor slow light using a double-tripod atom-light coupling scheme. The scheme involves three atomic ground states coupled to two excited states by six light fields. The oscillation due to the interaction between the two components was observed. On the basis of the stored light, our data showed that the double-tripod scheme behaves like the two outcomes of an interferometer enabling precision measurements of frequency detuning. We experimentally demonstrated a possible application of the double-tripod scheme as quantum memory/rotator for the two-colour qubit. Our study also suggests that the spinor slow light is a better method than a widely used scheme in the nonlinear frequency conversion.

\footnotetext{
${ }^{1}$ Department of Physics and Frontier Research Center on Fundamental and Applied Sciences of Matters, National Tsing Hua University, Hsinchu 30013, Taiwan. ${ }^{2}$ Institute of Theoretical Physics and Astronomy, Vilnius University, A. Goštauto 12, Vilnius 01108, Lithuania. Correspondence and requests for materials should be addressed to I.A.Y. (email: yu@phys.nthu.edu.tw).
} 
O ver the last decade there have been significant advances in studying the slow ${ }^{1-12}$, stored ${ }^{13-21}$ and stationary ${ }^{22-25}$ light stimulated by applications to low-light-level nonlinear optics ${ }^{26-35}$ and quantum information manipulation ${ }^{36-42}$. The slow and stationary light, forming due to the electromagnetically induced transparency (EIT) effect ${ }^{43-45}$, greatly enhance the light-matter interaction and enable nonlinear optical processes to achieve significant efficiency even at singlephoton level ${ }^{26-35}$. The storage of light using the dynamic EIT scheme transfers quantum states between photons and atoms, serving as quantum memory for photons ${ }^{38-42}$. The EIT-related research has made a great impact on the nonlinear optics and quantum information science ${ }^{26-42}$.

The two-component or spinor slow light (SSL) using a double tripod (DT) atom-light coupling scheme ${ }^{46-49}$ exhibits a number of additional distinct features. The SSL can lead to interesting phenomena, such as formation of the quasi-particles exhibiting Dirac spectra ${ }^{46,47}$ or oscillations between the two components ${ }^{47,48}$. It can also be exploited in designing novel photonic devices, for example, quantum memory/rotator for two-colour qubits, interferometers for sensitive measurements and high-efficiency media for nonlinear frequency conversion, as it will be discussed later in the article. Here we report the first experimental demonstration of the SSL.

Our experimental study of the SSL makes use of the DT transition scheme and was carried out with laser-cooled ${ }^{87} \mathrm{Rb}$ atoms. Details of the experimental setup can be found in the Methods section. The DT level scheme consists of three atomic ground states of $|0\rangle,|1\rangle$ and $|2\rangle$ and two excited states of $|A\rangle$ and $|B\rangle$, as depicted in Fig. 1a. One probe field (with the Rabi frequency $\varepsilon_{A}$ ) and two coupling fields (with the Rabi frequencies $\Omega_{A 1}$ and $\left.\Omega_{A 2}\right)$ drive the transitions from $|0\rangle,|1\rangle$ and $|2\rangle$ to $|A\rangle$, respectively, to form the first tripod configuration. Another probe field $\left(\varepsilon_{B}\right)$ and the other two coupling fields $\left(\Omega_{B 1}\right.$ and $\left.\Omega_{B 2}\right)$ drive the transitions from the same ground states to $|B\rangle$ to form the second tripod configuration. The DT scheme is a combination of two single-tripod schemes ${ }^{50-54}$, but its physics is more abundant due to the interaction between the two components of light coupled with two atomic coherences ${ }^{46-49}$.

In this work, we observe the oscillation between the two slow light components controlled by the two-photon detuning. In a proof-of-principle measurement, our data show that the DT scheme for the light storage behaves like the two outcomes of an interferometer enabling measurements of the frequency detuning with the precision of the order of $100 \mathrm{~Hz}$. Finally, we experimentally demonstrate a possible application of the DT scheme as quantum memory/rotator for the two-colour qubit, that is, the superposition state of two frequency modes.

\section{Results}

Theoretical background. In the DT system ${ }^{46-49}$, the dynamics of the two probe fields and two atomic coherences can be described by the Maxwell-Bloch equations ${ }^{46,49}$ :

$$
\begin{gathered}
\frac{1}{c} \frac{\partial}{\partial t}\left[\begin{array}{l}
\varepsilon_{A} \\
\varepsilon_{B}
\end{array}\right]+\frac{\partial}{\partial z}\left[\begin{array}{l}
\varepsilon_{A} \\
\varepsilon_{B}
\end{array}\right]=i \frac{\alpha \Gamma}{2 L}\left[\begin{array}{l}
\rho_{A} \\
\rho_{B}
\end{array}\right], \\
\frac{\partial}{\partial t}\left[\begin{array}{l}
\rho_{A} \\
\rho_{B}
\end{array}\right]=\frac{i}{2}\left[\begin{array}{l}
\varepsilon_{A} \\
\varepsilon_{B}
\end{array}\right]+\frac{i}{2}\left[\begin{array}{ll}
\Omega_{A 1} & \Omega_{A 2} \\
\Omega_{B 1} & \Omega_{B 2}
\end{array}\right]\left[\begin{array}{l}
\rho_{1} \\
\rho_{2}
\end{array}\right]-\frac{\Gamma}{2}\left[\begin{array}{l}
\rho_{A} \\
\rho_{B}
\end{array}\right], \\
\frac{\partial}{\partial t}\left[\begin{array}{l}
\rho_{1} \\
\rho_{2}
\end{array}\right]=\frac{i}{2}\left[\begin{array}{ll}
\Omega_{A 1}^{*} & \Omega_{B 1}^{*} \\
\Omega_{A 2}^{*} & \Omega_{B 2}^{*}
\end{array}\right]\left[\begin{array}{l}
\rho_{A} \\
\rho_{B}
\end{array}\right]+\left[\begin{array}{cc}
i \delta & 0 \\
0 & -i \delta
\end{array}\right]\left[\begin{array}{l}
\rho_{1} \\
\rho_{2}
\end{array}\right],
\end{gathered}
$$

where $\rho_{A}\left(\right.$ or $\rho_{B}$ ) is the optical coherence corresponding to the probe transition of $|0\rangle \rightarrow|A\rangle$ (or $|0\rangle \rightarrow|B\rangle), \rho_{1} \quad\left(\right.$ or $\left.\rho_{2}\right)$ is the ground-state coherence between $|0\rangle$ and $|1\rangle$ (or $|2\rangle$ ), $\Gamma$ is the spontaneous decay rate of the excited states, $\alpha$ is the optical density (OD) of the medium with the length $L$, and $\delta$ is the twophoton detuning, as illustrated in Fig. 1a. To reach the above equations, the probe fields are assumed to be much weaker than the coupling ones. In that case most atomic population is in the ground state $|0\rangle$, and one can treat the probe fields as a perturbation. All fast-oscillation exponential factors associating with center frequencies and wave vectors have been eliminated from the equations, and only slowly-varying amplitudes are retained.

To simplify the discussion, let the complex Rabi frequencies of the four coupling fields $\Omega_{n}=\Omega e^{i \theta_{n}}$ have the same constant amplitude of $\Omega$ and various phases $\theta_{n}$, where $n=A 1, A 2, B 1$ or $B 2$. We define $\theta \equiv\left(\theta_{A 1}-\theta_{A 2}\right)-\left(\theta_{B 1}-\theta_{B 2}\right)$ to be a relative phase among the four coupling fields. By changing $\theta$, one can substantially alter the dispersions and other properties of the two-component slow-light modes ${ }^{46-48}$. We will focus on the case where $\theta=\pi$. According to Equations (1)-(3), the relation between the input and output probe fields of the continuous wave is then given by (see Supplementary Note 1)

$$
\left[\begin{array}{c}
\varepsilon_{A, \text { out }} \\
\varepsilon_{B, \text { out }}
\end{array}\right]=\mathrm{e}^{-2 \phi^{2} / \alpha}\left[\begin{array}{cc}
\cos \phi & -\sin \phi \\
\sin \phi & \cos \phi
\end{array}\right]\left[\begin{array}{l}
\varepsilon_{A, \text { in }} \\
\varepsilon_{B, \text { in }}
\end{array}\right],
$$

with

$$
\phi=\frac{\alpha \Gamma}{2 \Omega^{2}} \delta .
$$

As only one probe field is present in the input, for example, $\varepsilon_{A, \text { in }}=1$ and $\varepsilon_{B, \text { in }}=0$, the probe transmissions are

$$
\left[\begin{array}{c}
\left|\varepsilon_{A, \text { out }}\right|^{2} \\
\left|\varepsilon_{B, \text { out }}\right|^{2}
\end{array}\right]=\mathrm{e}^{-4 \phi^{2} / \alpha}\left[\begin{array}{c}
\cos ^{2} \phi \\
\sin ^{2} \phi
\end{array}\right] \text {. }
$$

Thus oscillations between the two modes show up at the output of the medium.

The oscillation phenomenon as well as the interaction of the probe fields with the atomic medium is determined by the four coupling fields. Figure $1 \mathrm{~b}$ illustrates a physical picture of the role played by the coupling fields for the case where $\theta=\pi$. The coupling fields couple the excited state $|A\rangle(|B\rangle)$ to a symmetric (antisymmetric) superposition of the atomic ground states $|1\rangle$ and $|2\rangle$ labelled by $\left|1^{\prime}\right\rangle\left(\left|2^{\prime}\right\rangle\right)$. Supplementary Note 2 explains in detail how the DT system is equivalent to the two coupled $\Lambda$ systems. The detuning $\delta$ introduces the coupling between the newly defined ground states $\left|1^{\prime}\right\rangle$ and $\left|2^{\prime}\right\rangle$, as one can see in Fig. 1b. This leads to the interaction between the probe fields and hence to their oscillations during the propagation in the atomic medium.

The SSL oscillations can also be observed for the relative phase $\theta$ of coupling fields other than $\pi$. However, at $\theta=0$ the excited states $|A\rangle$ and $|B\rangle$ are coupled to the same (symmetric) superposition of $|1\rangle$ and $|2\rangle$ (see Supplementary Note 3 and Supplementary Fig. 1). In that case, the DT system becomes equivalent to the double- $\Lambda$ system, and no oscillation can occur between the probe fields. Larger $\theta$ makes the oscillations more prominent, and $\theta=\pi$ gives the maximum contrast or difference between two output probe fields at a small $\delta$ (see Supplementary Note 4 and Supplementary Fig. 2). For this reason, $\theta=\pi$ was chosen in the experiment.

According to equations (5) and (6), the detuning $\delta$ not only causes the probe fields to oscillate, but also reduces the total output energy. In the EIT spectrum, the two-photon resonance (that is, a condition where the frequency difference between the probe and coupling fields is equal to that between two ground states driven by them) corresponds to the transparency peak or the maximum transmission. A larger two-photon detuning makes less transmission. In the present EIT system, the two-photon detuning and coupling Rabi frequency correspond to $\delta$ and $\sqrt{2} \Omega$. On the basis of equation (27) in ref. 45, the transmission around 


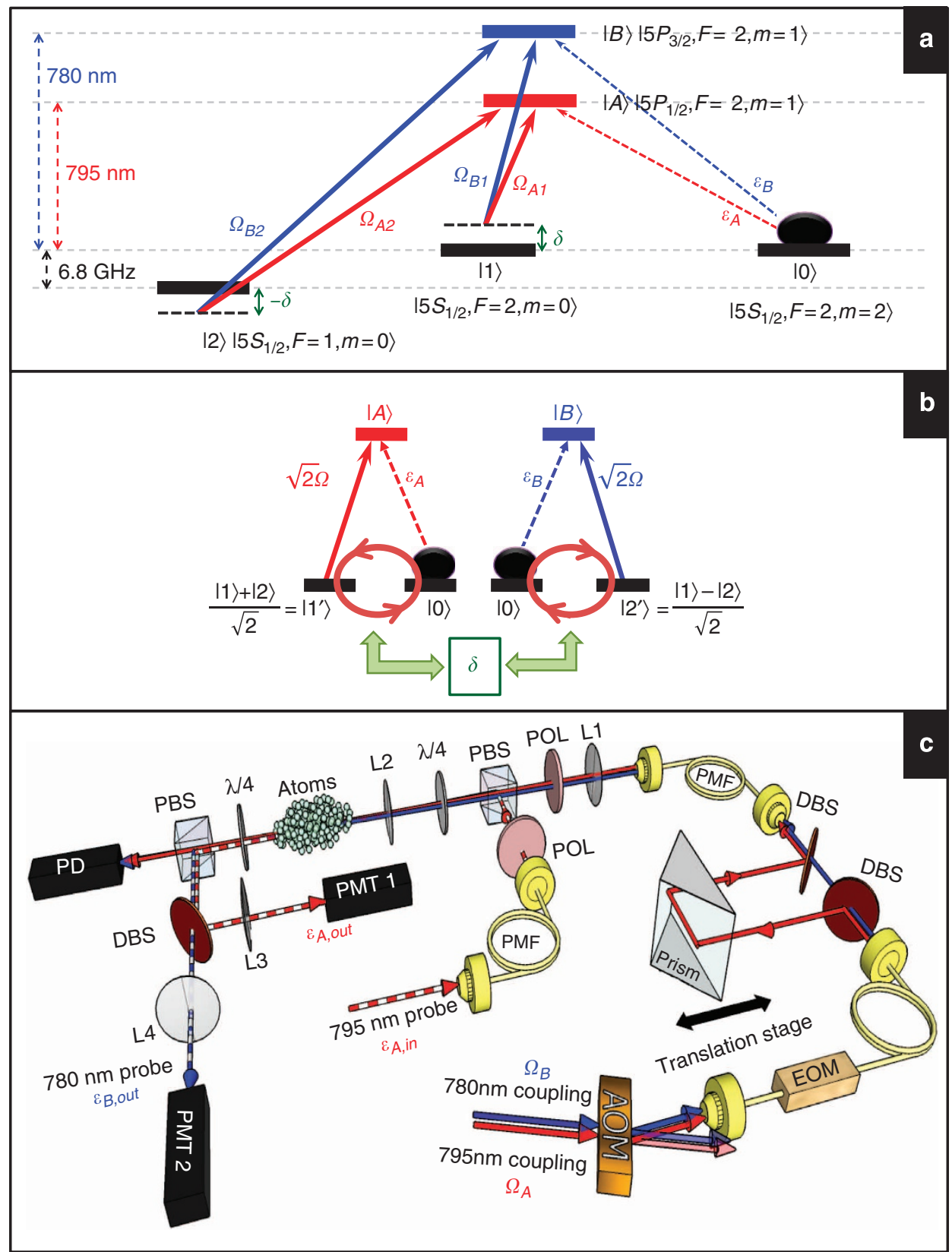

Figure 1 | Transition diagram and experimental setup. (a) Relevant energy levels and laser excitations in the double-tripod (DT) system for ${ }^{87} \mathrm{Rb}$ atoms. Here $\varepsilon_{A}$ and $\varepsilon_{B}$ represent the probe fields; $\Omega_{A 1}, \Omega_{A 2}, \Omega_{B 1}$ and $\Omega_{B 2}$ indicate the coupling fields; $\delta$ (or $-\delta$ ) is the two-photon detuning with respect to the Raman transition between $|0\rangle$ and $|1\rangle$ (or $|2\rangle$ ). (b) Two coupled $\Lambda$ systems with an effective Rabi frequency $\sqrt{2} \Omega$. The schema is equivalent to the DT system with Rabi frequencies of the four coupling fields having the same amplitude $\Omega$ and relative phase $\theta=\pi$. The two-photon detuning $\delta$ of the DT system introduces coupling between the effective $\Lambda$ systems. (c) Schematic experimental setup. AOM, acousto-optic modulator; DBS, dichroic beam splitter; EOM, electro-optic modulator; L1-L4, lenses with focal lengths of 300, 200, 300 and 500 mm, respectively; PBS, polarizing beam splitter; PD, photo detector; PMF, polarization-maintained optical fibre; PMT, photo multiplier; POL, polarizer; $\lambda / 4$, quarter-wave plate.

the EIT peak is given by $\exp \left[-4 \alpha \delta^{2} \Gamma^{2} /(\sqrt{2} \Omega)^{4}\right]$, which is exactly the exponential decay term in equation (6).

Nonlinear frequency conversion. By varying one of the three factors, the two-photon detuning $\delta$, optical density (OD) $\alpha$ and coupling Rabi frequency $\Omega$, while keeping the other two fixed, we can make the two slow-light outputs oscillate alternatively. The oscillations are accompanied by the decay of the total output energy, since the EIT is degraded due to the detuning $\delta$. Nevertheless, a large OD can significantly reduce the decay over an oscillation period. Regarding the nonlinear optical process that converts light from one frequency to another, one can use the DT scheme at $\phi=\pi / 2$. According to equation (6), an OD of 250 enables the conversion efficiency of $\sim 96 \%$. The same efficiency requires an OD of 500 in the widely used double- $\Lambda$ scheme ${ }^{55}$. Hence, the DT scheme is a new and advantageous method of nonlinear frequency conversion.

Oscillation of spinor slow light. We first demonstrated the oscillation phenomenon resulting from the SSL in the DT scheme. As only one probe pulse $\left(\varepsilon_{A}\right)$ was sent to the input and the four coupling fields were constantly present, the outputs of 
both probe pulses were measured at different two-photon detunings. Figure $2 \mathrm{a}$, b show the two output powers as functions of time at $\delta=0$ and $\delta=2 \pi \times 160 \mathrm{kHz}$. At $\delta=0, \varepsilon_{B}$ will be generated by $\varepsilon_{A}$ if $\theta \neq \pi$ and, $\varepsilon_{B}$ will not be generated if $\theta=\pi$ (see Supplementary Note 5 and Supplementary Fig. 3). Because of this condition, we were able to properly set $\theta=\pi$ by minimizing the output of $\varepsilon_{B}$. The energy transmissions of $\varepsilon_{A}$ and $\varepsilon_{B}$ as functions of $\delta$ are shown in Fig. 2c. Two outputs oscillate alternatively; when one reaches minima, the other becomes maxima and vice versa. Their total transmitted energy decays as $|\delta|$ increases, because the detuning away from the EIT resonance is associated with the losses. The oscillation phenomenon behaves qualitatively as described by equations (5) and (6).

Quantitatively, we compare the data with the predictions by numerically solving equations (1)-(3) as shown in Fig. $2 \mathrm{a}-\mathrm{c}$. To better describe the experimental condition, we also considered the phase mismatch $\Delta_{k}$ and the dephasing rates $\gamma_{1}$ and $\gamma_{2}$ of the ground-state coherences $\rho_{1}$ and $\rho_{2}$ in the calculation. The following two terms

$$
\left[\begin{array}{cc}
i \Delta_{k} / 2 & 0 \\
0 & -i \Delta_{k} / 2
\end{array}\right]\left[\begin{array}{l}
\varepsilon_{A} \\
\varepsilon_{B}
\end{array}\right] \text { and }\left[\begin{array}{cc}
-\gamma_{1} & 0 \\
0 & -\gamma_{2}
\end{array}\right]\left[\begin{array}{l}
\rho_{1} \\
\rho_{2}
\end{array}\right]
$$

have been added to the left-hand side of equation (1) and the right-hand side of equation (3), respectively. In the first term, $\Delta_{k} \equiv\left(-\overrightarrow{\mathbf{k}}_{p A}+\overrightarrow{\mathbf{k}}_{c A}-\overrightarrow{\mathbf{k}}_{c B}+\overrightarrow{\mathbf{k}}_{p B}\right) \cdot \hat{\mathbf{z}}$ describes the effect of phase mismatch ${ }^{56}$, where $\overrightarrow{\mathbf{k}}_{p A}$ and $\overrightarrow{\mathbf{k}}_{p B}$ are the wave vectors for the probe
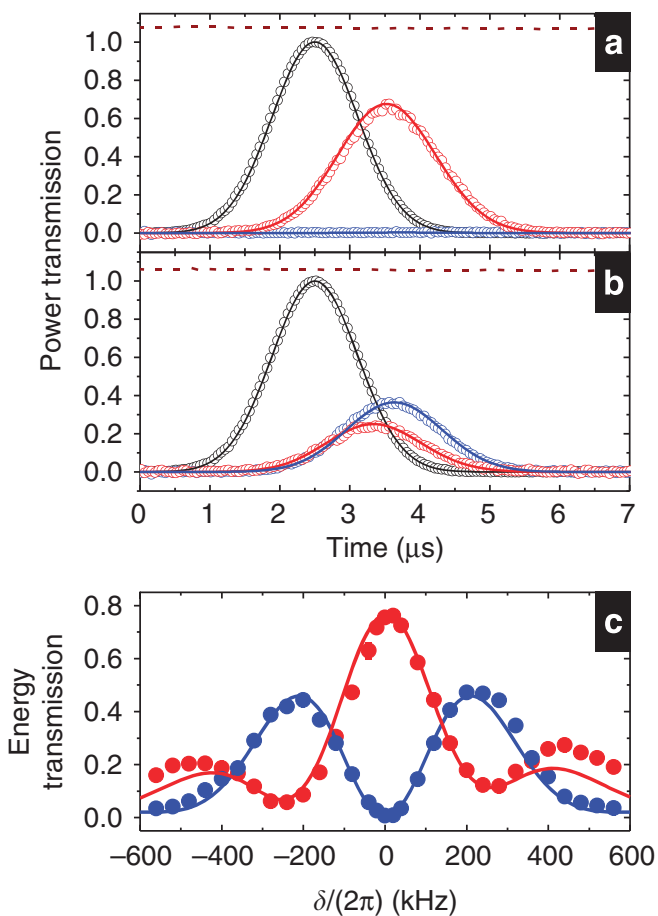

Figure 2 | Oscillation phenomenon in the double-tripod scheme.

$(\mathbf{a}, \mathbf{b})$ The output powers of $\varepsilon_{A}$ and $\varepsilon_{B}$ versus time for $\varepsilon_{A}$ being the only input, where $\delta=0$ in (a) and $2 \pi \times 160 \mathrm{kHz}$ in (b). Black and red circles are experimental data of the input and output $\varepsilon_{A}$; blue circles are those of the output $\varepsilon_{B}$. Dashed lines represent data of the four coupling fields. Solid lines are theoretical predictions. (c) Energy transmissions of the two probes as functions of $\delta$. Circles are experimental data and solid lines are theoretical predictions. In the theoretical calculation, $\alpha=20, \Omega_{A 1}=\Omega_{A 2}=$ $\Omega_{B 1}=\Omega_{B 2}=0.51 \Gamma, \gamma_{1}=0, \gamma_{2}=3.7 \times 10^{-3} \Gamma$ and $\Delta_{k} L=0.6$, where $\Gamma=2 \pi \times 6 \mathrm{MHz}$. These parameters were determined by two ordinary (single- $\Lambda$ ) EIT and two double- $\Lambda$ measurements. Error bars are about the size of data points. fields $\varepsilon_{A}$ and $\varepsilon_{B}$, and $\overrightarrow{\mathbf{k}}_{c A}$ and $\overrightarrow{\mathbf{k}}_{c B}$ are those for the coupling fields $\Omega_{A 1}$ and $\Omega_{B 1}$ (or $\Omega_{A 2}$ and $\Omega_{B 2}$ ). There are no free parameters in the calculation, in which all parameters were determined by two ordinary (single- $\Lambda$ ) EIT and two double- $\Lambda$ measurements (see the Methods section). In Fig. $2 c, \varepsilon_{A}$ 's minima are not completely zero, and the probe transmission is slightly asymmetric for the positive and negative detunings. The non-zero minima are caused by $\Delta_{k} \neq 0$ and the finite frequency bandwidth of the input probe pulse, whereas the asymmetry results from the combination of $\Delta_{k} \neq 0$ and $\gamma_{1} \neq \gamma_{2}$ (see Supplementary Note 6 and Supplementary Fig. 4 for more details). For $|\delta|<300 \mathrm{kHz}$, discrepancies between the data and predictions are comparable to the measurement uncertainty, which is about the size of the data point. At a large detuning, some data point can deviate from the theoretical line significantly more than the uncertainty. The deviation may be due to the one-photon detunings of the 795 and $780 \mathrm{~nm}$ transitions, which cannot be determined accurately and has not been taken into account in the calculation.

Spinor-slow-light interferometer. The number of oscillation cycles can be considerably increased with the storage and retrieval of SSL. The idea is based on the intuition that the propagation time of the light pulses in the medium is equivalent to the storage time of motionless ones transformed into the atomic coherences $\rho_{1}$ and $\rho_{2}$. In equation (5), the quantity $t_{d} \equiv \alpha \Gamma /\left(2 \Omega^{2}\right)$, representing the SSL propagation delay time, determines the mixing angle $\phi$ $\left(=t_{d} \delta\right.$ ) between the two slow-light components. If the slow light is stored for a time $t_{s} \gg t_{d}$, the propagation time $t_{d}$ is to be replaced by the storage time $t_{s}$ in the phase of the SSL oscillation

$$
\phi=t_{s} \delta
$$

without introducing extra losses. In this work, the propagation delay time $t_{d}$ was merely around $1 \mu \mathrm{s}$. With the storage time $t_{s}$ considerably longer than $1 \mu \mathrm{s}$, a small two-photon detuning (compared with the EIT bandwidth) can still result in a large oscillation phase. Figure 3a clearly demonstrates the above idea and verifies equation (7) at $t_{s}=15 \mu \mathrm{s}$. The data of retrieved energies versus $\delta$ exhibit more than three oscillation cycles and show no oscillation amplitude attenuation. Both $\varepsilon_{A}$ 's and $\varepsilon_{B}$ 's minima with values of $0.017 \pm 0.012$ are close to zero. The data of positive and negative values of $\delta$ are very symmetric except a small shift of the origin in $\delta$. This shift is due to the a.c. Stark effect induced by the coupling fields not present during the light storage. The data in Fig. 3a behave just like $\cos ^{2} \phi$ and $\sin ^{2} \phi$, where $\phi=\left(t_{s}+t_{d}\right) \delta+\phi_{0}$. Their best fits determine that the oscillation period is $2 \pi \times(30.8 \pm 0.1) \mathrm{kHz}$. This period indicates $t_{s}+t_{d} \approx 16 \mu \mathrm{s}$, which is in agreement with the actual value in the measurement.

The data of two output probe energies in Fig. 3a are similar to the two outcomes of an interferometer. According to equation (7), one can precisely determine the two-photon detuning $\delta$ with such DT interferometer. The decay time constant of stored light in this experiment was $\sim 76 \mu \mathrm{s}$. Utilizing this sufficiently long time constant, we demonstrated a proof-ofprinciple measurement in Fig. 3b,c. Data in each figure were taken under a fixed $\delta$. We set the difference in the $\delta$ to be $2 \pi \times 10.0 \mathrm{kHz}$ for the two figures. Note that the difference of the two values can be set much more accurately than their absolute values. We measured the retrieved energies against the storage time as shown in the figures. The best fits of the data determine the oscillation periods $\left(T_{s}\right)$ of $49.9 \pm 0.3$ and $25.3 \pm 0.1 \mu \mathrm{s}$. On the basis of equation (7), we have $\delta=\pi / T_{s}$, which gives the difference of the two $\delta$ s equal to $2 \pi \times 9.7 \mathrm{kHz}$. The measured value of the difference is consistent with the actual one, showing that the light-storage DT scheme can be used to determine the detuning $\delta$ or anything that can affect $\delta$, such as light shifts, Zeeman shifts, 

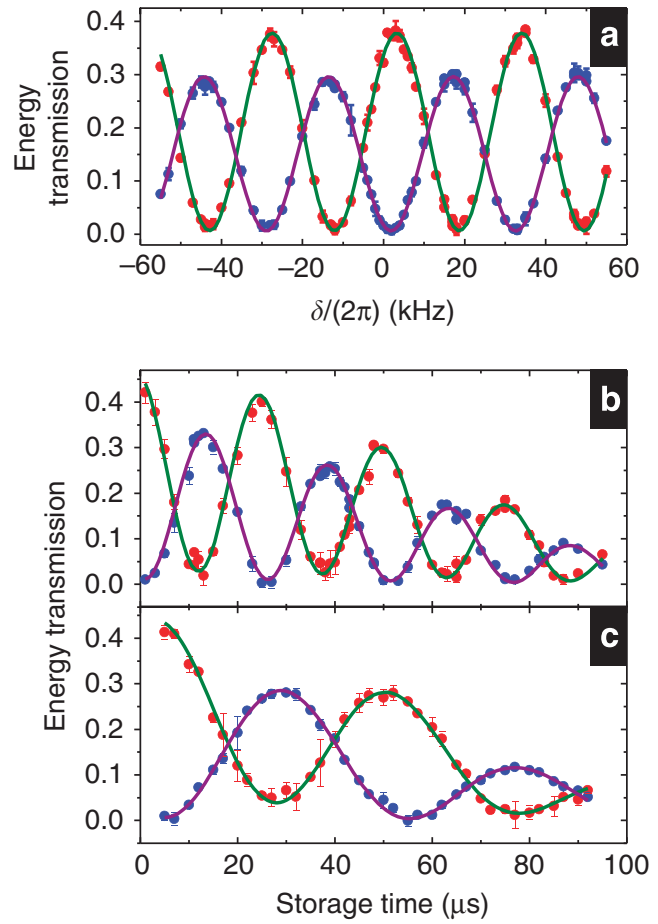

Figure 3 | Light storage in the double-tripod scheme with the probe $\varepsilon_{A}$ being the only input. (a) After a storage time of $15 \mu \mathrm{s}$, retrieved energies of $\varepsilon_{A}$ (red circles) and $\varepsilon_{B}$ (blue circles) as functions of $\delta$. Green and purple lines are the best fits, determining the oscillation period equal to $2 \pi \times(30.8 \pm 0.1) \mathrm{kHz}$. (b,c) Retrieved energies as functions of the storage time at two values of $\delta$ differing by $2 \pi \times 10.0 \mathrm{kHz}$. The best fits in $\mathbf{b}$ (or $\mathbf{c}$ ) determine the oscillation period equal to $49.9 \pm 0.3$ (or $25.3 \pm 0.1$ ) $\mu$ s and the decay time constant equal to $76.8 \pm 1.2$ (or $75.8 \pm 1.5$ ) $\mu$ s.

and so on. The precision demonstrated here is of the order of $100 \mathrm{~Hz}$.

Two-colour qubits. The single-photon SSL can be considered as the qubit with the superposition state of two frequency modes or, simply, as the two-colour qubit. Its wave function is given by

$$
|\psi\rangle=a\left|1_{\omega_{A}}, 0_{\omega_{B}}\right\rangle+b\left|0_{\omega_{A}}, 1_{\omega_{B}}\right\rangle,
$$

where $a$ and $b$ are the probability amplitudes with the constraint of $|a|^{2}+|b|^{2}=1$. Such a qubit can be produced by sending a single photon to the DT system. On the other hand, the DT system can be used as quantum memory/rotator for the qubits. In the classical limit, the above wave function corresponds to $a \vec{\varepsilon}_{A}+b \vec{\varepsilon}_{B}$, where $\vec{\varepsilon}_{A}$ and $\vec{\varepsilon}_{B}$ represent the basis or electric fields of the two frequency modes. Considering $(a, b)=(\cos \phi, \sin \phi)$, the generation of this wave function has been demonstrated in Fig. 3a.

To see whether the light storage in the DT system can preserve $(a, b)$, we simultaneously sent both $\varepsilon_{A}$ and $\varepsilon_{B}$ pulses to the medium and made $\delta=0$ during the storage. Figure $4 \mathrm{a}-\mathrm{c}$ show the temporal profiles of the two retrieved pulses at different ratios of $a$ to $b$. In each figure, the shapes of the two retrieved pulses and their energy ratio after the storage time of $3 \mu$ s are very close to those after the storage time of $33 \mu \mathrm{s}$. Furthermore, one should be able to utilize a two-photon detuning applied during the storage to change $\phi$ or the $a$-to- $b$ ratio. The detuning is essentially the Zeeman or a.c. Stark shift induced by a pulse of magnetic field or by a far detuned microwave or laser pulse. The amount of the change is controlled by the product of the detuning and pulse duration as shown by the data in Fig. 3. Although our

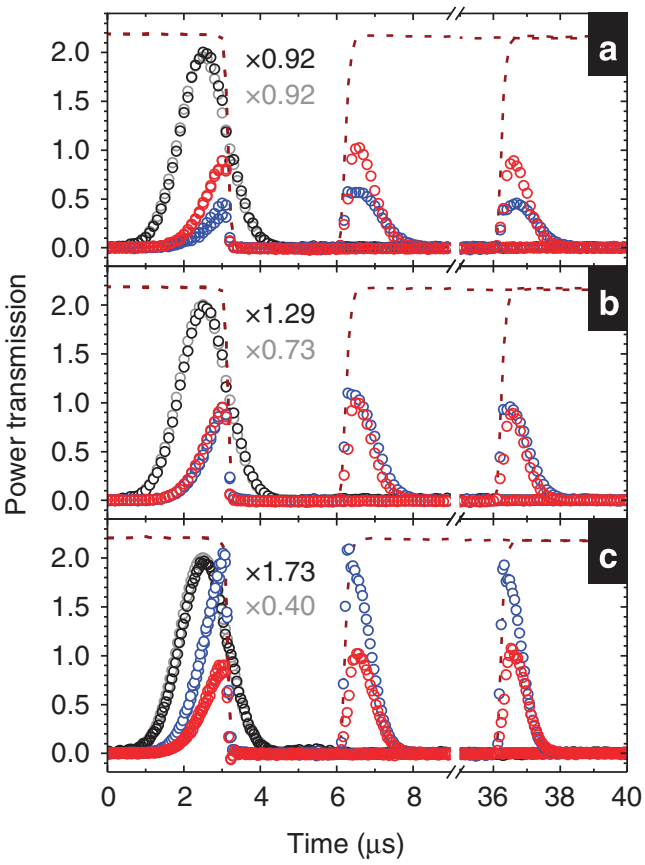

Figure 4 | Storage and retrieval of two-colour light pulses in the doubletripod scheme. The detuning $\delta$ was set to zero during the storage time. Black and grey circles are the input $\varepsilon_{A}$ and $\varepsilon_{B}$ pulses scaled up or down by the factors shown in the plots; red and blue circles are the two retrieved pulses after storage times of 3 and $33 \mu \mathrm{s}$. In $\mathbf{a}, \mathbf{b}$ and $\mathbf{c}$, energy ratios of the two retrieved pulses after the storage time of $3(33) \mu$ sare 1.5 (1.5), $0.84(0.92)$ and $0.55(0.52)$, respectively.

demonstrations were done with classical light, the results suggest that the light-storage DT scheme can be used as the quantum memory/rotator for two-colour qubits.

\section{Discussion}

We observed the two-component slow light or SSL in the DT system as demonstrated by the oscillation of the two output light pulses. The oscillations are induced by the coupling between the two ground-state coherences due to the non-zero detuning $\delta$, as shown in Fig. 1b. The data of the light-storage DT scheme were used to determine the two-photon detuning (or anything that can affect the detuning such as light shifts, Zeeman shifts, and so on) in the system with the satisfactory accuracy and precision. The sensitivity of our method is based on the fact that the light is stored in a superposition of two atomic levels, that is, in two atomic coherences. Therefore, for sufficiently long storage times, even a slight energy mismatch between these levels can lead to a large accumulated phase which can be detected by measuring the conversion of the regenerated light into another component. The storage time and the measurement precision in this work were of the orders of $100 \mu \mathrm{s}$ and $100 \mathrm{~Hz}$, respectively. In ref. 19, an optical dipole trap was used to confine cold atoms, leading to the lightstorage time of $\sim 1 \mathrm{~s}$. In principle, a longer storage time can result in a better precision of the measured frequency.

The two-colour qubit is a superposition state of two frequency modes. We demonstrated a possible application of employing the DT scheme as quantum memory/rotator for the two-colour qubits by utilizing a two-photon detuning during the storage time. Furthermore, the SSL may lead to interesting physics such as spinor Bose-Einstein condensation of dark-state polaritons ${ }^{23}$ and Dirac particles ${ }^{46,47}$. It can also be used to achieve high conversion efficiencies in the nonlinear frequency conversion. 
This work is the first experiment of the SSL research, which may result in novel applications in quantum information manipulation, precision measurement and nonlinear optics.

Finally, we note that the current DT setup is arranged in the co-propagation configuration in which the four coupling fields propagate in the same direction and completely overlap. The phase match condition can be closely achieved in the copropagation configuration. One can also use the counterpropagation configuration to form the $\mathrm{SSL}^{46,47}$. In such a situation, the coupling fields come from two opposite directions to interact with atoms. Yet a phase mismatch can be significant even at an optimum alignment of laser beams, which has been already seen in our previous experiments utilizing the counterpropagating configuration for other studies ${ }^{20,30}$. This may represent an obstacle in the SSL formation for the counterpropagating configuration. Regarding nonlinear frequency conversion, the co-propagation scheme ${ }^{48}$ allows for a higher efficiency as compared with the counter-propagating one $\mathrm{e}^{47}$.

\section{Methods}

Experimental setup. A cigar-shaped cloud of cold ${ }^{87} \mathrm{Rb}$ atoms with the dimension of $\sim 9 \times 2 \times 2 \mathrm{~mm}^{3}$ was produced by a magneto-optical trap (MOT) ${ }^{57}$. Typically, we can trap $10^{9}$ atoms with a temperature of about $300 \mu \mathrm{K}$ in the MOT. Figure 1a shows the relevant energy levels and laser excitations in the experiment. The system of cold atoms is not a necessary condition for the SSL formation. Yet utilizing cold atoms here helps to minimize losses such as those induced by collisional and transit decoherence processes. In that case, the experimental system can be as simple as the system in the theoretical model, these decoherence processes are negligible, and the alignment of the laser beams is more flexible.

To make a DT system as simple as possible, we put all population to a single Zeeman state. This can be achieved with strong $\sigma+$ laser fields driving the transitions from the ground states $|F=1\rangle$ and $|F=2\rangle$ to the excited states $\left|F^{\prime}=2\right\rangle$ and $\left|F^{\prime \prime}=2\right\rangle$, where $F, F^{\prime}$ and $F^{\prime \prime}$ denote the hyperfine states in the $\left|5 S_{1 / 2}\right\rangle,\left|5 P_{1 / 2}\right\rangle$ and $\left|5 P_{3 / 2}\right\rangle$ energy levels, respectively. Hence, the Zeeman state $|F=2, m=2\rangle$ was the only dark ground state in the system. All population can be optically pumped to it. The two probe fields drove the transitions from $|F=2\rangle$ to $\left|F^{\prime}=2\right\rangle$ and $\left|F^{\prime \prime}=2\right\rangle$ with the $\sigma-$ polarization. Because all the ground Zeeman states other than $|F=2, m=2\rangle$ had no population, only the probe transitions from $|F=2, m=2\rangle$ to $\left|F^{\prime}=2, m=1\right\rangle$ and $\left|F^{\prime \prime}=2, m=1\right\rangle$ were relevant. Consequently, the entire atom-light coupling scheme becomes a simple DT system as shown in Fig. 1a, and all other states and transitions are irrelevant.

The probe field of $\varepsilon_{A}\left(\varepsilon_{B}\right)$ and the two coupling fields of $\Omega_{A 1}$ and $\Omega_{A 2}\left(\Omega_{B 1}\right.$ and $\Omega_{B 2}$ ) had wavelengths of $\sim 795 \mathrm{~nm}(780 \mathrm{~nm})$. The spontaneous decay rate of the excited states, $\Gamma$, is $2 \pi \times 6 \mathrm{MHz}$. Figure 1c shows the schematic experimental setup. We first overlapped $\Omega_{A 1}$ and $\Omega_{B 1}$, and sent them through an acousto-optic modulator and an electro-optic modulator (EOM). The EOM generated $\Omega_{A 2}$ and $\Omega_{B 2}$. We set the two-photon detunings $\delta$ and $-\delta$ asymmetrically by changing the operation frequencies of EOM and acousto-optic modulator.

After coming out of the EOM, the two $795 \mathrm{~nm}$ coupling fields and the two $780 \mathrm{~nm}$ ones were separated, propagated through different paths and then were overlapped again. We tuned the difference of the two path lengths to ensure that $\theta=\pi$. Propagating along the major axis of the atom cloud, the probe beams were focused to an $e^{-2}$ full width of $150 \mu \mathrm{m}$. The coupling beams had a much larger size to cover the entire atom cloud. We set the separation angle between the propagation directions of the probe and coupling beams to $\sim 0.35^{\circ}$. The input probe pulse has the Gaussian shape with an $e^{-2}$ full width of $2.5 \mu$ s and a peak power of $15 \mathrm{nW}$. Other details of the experimental setup are similar to those in refs 20,30 dealing with the EIT-based slow and stationary light for $\Lambda$ or double- $\Lambda$ systems involving single atomic ground-state coherences.

Experimental procedure and timing sequence. Before each measurement, we first reduced the MOT repumping field intensity from 1.1 to $0.0044 \mathrm{~mW} \mathrm{~cm}^{-2}$ for $\sim 7 \mathrm{~ms}$. This can increase the atomic density and, thus, the OD of the system. Then, we switched off the magnetic, repumping and trapping fields of the MOT at $t=-718,-74$ and $-48 \mu \mathrm{s}$, respectively $(t=0$ denoted as the time of the input probe pulse peak). An optical pumping field was used during the period from $t=-74$ to $-18 \mu \mathrm{s}$, driving the transition from $|F=1\rangle$ to $\left|F^{\prime \prime}=2\right\rangle$ with an intensity of $5 \mathrm{~mW} \mathrm{~cm}^{-2}$ and the $\sigma+$ polarization. The coupling fields $\Omega_{A 1}$ and $\Omega_{B 1}$ with intensities of 98 and $12 \mathrm{~mW} \mathrm{~cm}{ }^{-2}$ were switched on at $t=-78 \mu \mathrm{s}$. This optical pumping field together with the coupling fields $\Omega_{A 1}$ and $\Omega_{B 1}$ optically pumped all population to the Zeeman state of $|F=2, m=2\rangle$. At $t=-5 \mu \mathrm{s}$, we reduced $\Omega_{A 1}$ and $\Omega_{B 1}$ to the designated experimental value and switched on $\Omega_{A 2}$ and $\Omega_{B 2}$ with the same value. After the four coupling fields for the SSL were present, we fired the probe pulse $\varepsilon_{A}$. Two photo multipliers (Hamamatsu PMT H6780-20 and H10720) were used to detect the signals of the two output probe pulses $\varepsilon_{A}$ and $\varepsilon_{B}$. Signals from the PMTs were sent to a digital oscilloscope (Agilent MSO6014A). Data were averaged 200 times by the oscilloscope before being acquired by a computer. After the measurement was complete, we turned off the coupling fields and turned back on the MOT. The above measurement sequence was repeated every $0.15 \mathrm{~s}$.

Determination of experimental parameters. With two single- $\Lambda$ and two double$\Lambda$ measurements, we determined the experimental parameters of the optical density $(\alpha)$, coupling Rabi frequencies $\left(\Omega_{A 1}, \Omega_{A 2}, \Omega_{B 1}\right.$ and $\left.\Omega_{B 2}\right)$, dephasing rates of the ground-state coherences $\left(\gamma_{1}\right.$ and $\left.\gamma_{2}\right)$ and degree of phase mismatch $\left(\Delta_{k} L\right)$. The procedure is described below. We first measured the slow light output in the single$\Lambda$ system formed by $|0\rangle,|1\rangle$ and $|A\rangle$. During the measurement, the coupling field of $\Omega_{A 1}$ was constantly present. The experimental data are shown in Fig. 5a. Varying the parameters of $\alpha, \Omega_{A 1}$ and $\gamma_{1}$ in the numerical calculation, we fitted the data with the theoretical prediction. Note that $\Delta_{k}$ has no role in the output amplitude of the probe pulse in the single- $\Lambda$ system. The best fit determined the experimental parameters of $\alpha, \Omega_{A 1}$ and $\gamma_{1}$.

Similarly, we measured the slow light output in another EIT system formed by $|0\rangle,|2\rangle$ and $|A\rangle$ to determine $\alpha, \Omega_{A 2}$ and $\gamma_{2}$. The experimental data are shown in
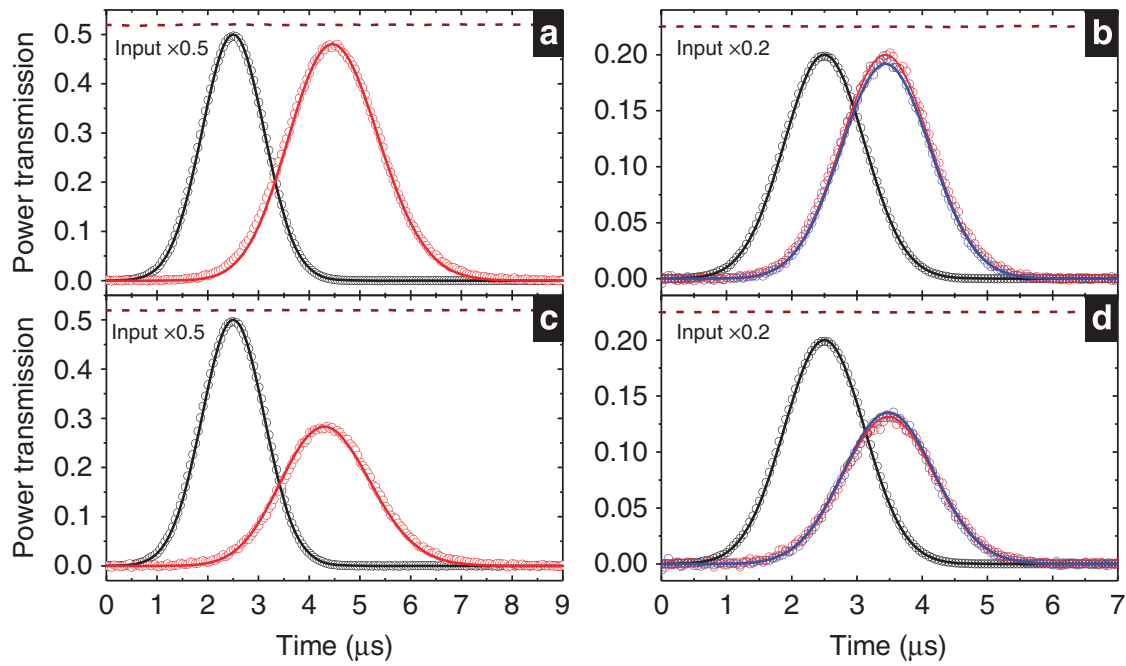

Figure 5 | Determination of experimental parameters. (a,c) The slow light output of $\varepsilon_{A}$ under the coupling field of $\Omega_{A 1}$ (or $\Omega_{A 2}$ ) in the ordinary (single- $\Lambda$ ) EIT scheme. (b,d) The slow light outputs of $\varepsilon_{A}$ and $\varepsilon_{B}$ under the coupling fields of $\Omega_{A 1}$ and $\Omega_{B 1}$ (or $\Omega_{A 2}$ and $\Omega_{B 2}$ ) in the double- $\Lambda$ scheme with $\varepsilon_{A}$ being the only input. Black and red circles are the experimental data of the input and output of $\varepsilon_{A}$; blue circles are those of the output of $\varepsilon_{B}$; dashed lines represent the data of the coupling field timing; solid lines are the theoretical predictions. In the theoretical calculation, $\theta=\pi, \alpha=20 \pm 1$, $\left|\Omega_{A 1}\right|=\left|\Omega_{A 2}\right|=(0.51 \pm 0.02) \Gamma,\left|\Omega_{B 1}\right|=\left|\Omega_{B 2}\right|=(0.52 \pm 0.015) \Gamma, \gamma_{1} \leq 3 \times 10^{-4} \Gamma$ or is effectively $0, \gamma_{2}=3.7 \times 10^{-3} \Gamma$ and $\Delta_{k} L=0.6$. 
Fig. 5c. We imposed a constraint that $\alpha$ values in the two EIT systems can only differ within the uncertainty. The determined $\gamma_{2}$ is rather large as compared with the dephasing rate in our previous work ${ }^{20}$. This $\gamma_{2}$ of $3.7 \times 10^{-3} \Gamma$ corresponds to a coherence time of $3.6 \mu \mathrm{s}$. Because the data of light storage in this work still showed a reasonably good coherence time of $76 \mu$ s as compared with the previous work, this large $\gamma_{2}$ might be due to the new fibre-based electro-optic modulator (EOM) used in the experiment. The EOM produced the sidebands, which served as the coupling fields of $\Omega_{A 2}$ and $\Omega_{B 2}$ (see Fig. 1c).

After $\alpha, \Omega_{A 1}$ and $\gamma_{1}$ were determined, we kept $\Omega_{A 1}$ and the experimental condition unchanged, and performed the measurement of the double- $\Lambda$ system formed by $|0\rangle,|1\rangle,|A\rangle$ and $|B\rangle$. With $\varepsilon_{A}$ being the only input, Fig. 5b shows the experimental data of the two output probe pulses. We fitted the data with the predictions, and the best fit determined $\Omega_{B 1}$ and $\Delta_{k} L$. Note that the same product of $\Delta_{k}$ and $L$ results in the same calculation result. Similarly, we measured two output probe pulses in another double- $\Lambda$ system formed by $|0\rangle,|2\rangle,|A\rangle$ and $|B\rangle$ to determine $\Omega_{B 2}$ and $\Delta_{k} L$. The experimental data are shown in Fig. $5 \mathrm{~d}$. The $\Delta_{k} L$ values determined from the two double- $\Lambda$ systems were about the same. The experimental condition was set and determined by the above procedure. We kept the condition unchanged and measured the data of the DT system. The determined parameters were used to calculate the theoretical predictions as shown in Fig. 2a-c.

\section{References}

1. Hau, L. V., Harris, S. E., Dutton, Z. \& Behroozi, C. H. Light speed reduction to 17 meters per second in an ultracold atomic gas. Nature 397, 594-598 (1999).

2. Kash, M. M. et al. Ultraslow group velocity and enhanced nonlinear optical effects in a coherently driven hot atomic gas. Phys. Rev. Lett. 82, 5229-5232 (1999).

3. Bigelow, M. S., Lepeshkin, N. N. \& Boyd, R. W. Observation of ultraslow light propagation in a ruby crystal at room temperature. Phys. Rev. Lett. 90, 113903 (2003).

4. Okawachi, Y. et al. Tunable all-optical delays via Brillouin slow light in an optical fiber. Phys. Rev. Lett. 94, 153902 (2005).

5. Novikova, I., Phillips, D. F. \& Walsworth, R. L. Slow light with integrated gain and large pulse delay. Phys. Rev. Lett. 99, 173604 (2007).

6. Krauss, T. F. Why do we need slow light? Nat. Photon. 2, 448-450 (2008)

7. Siddons, P., Bell, N. C., Cai, Y., Adams, C. S. \& Hughe, I. G. A gigahertzbandwidth atomic probe based on the slow-light Faraday effect. Nat. Photon. 3, 225-229 (2009).

8. Khurgin, J. B. Slow light in various media: a tutorial. Adv. Opt. Photon. 2, 287-318 (2010)

9. Wu, C., Khanikaev, A. B. \& Shvets, G. Broadband slow light metamaterial based on a double-continuum Fano resonance. Phys. Rev. Lett. 106, 107403 (2011).

10. Capmany, J., Gasulla, I. \& Sales, S. Harnessing slow light. Nat. Photon. 5, 731733 (2011).

11. Grinberg, P. et al. Nanocavity linewidth narrowing and group delay enhancement by slow light propagation and nonlinear effects. Phys. Rev. Lett. 109, 113903 (2012).

12. Muñoz, M. C. et al. Optically induced indirect photonic transitions in a slow light photonic crystal waveguide. Phys. Rev. Lett. 112, 053904 (2014).

13. Fleischhauer, M. \& Lukin, M. D. Dark-state polaritons in electromagnetically induced transparency. Phys. Rev. Lett. 84, 5094-5097 (2000).

14. Liu, C., Dutton, Z., Behroozi, C. H. \& Hau, L. V. Observation of coherent optical information storage in an atomic medium using halted light pulses. Nature 409, 490-493 (2001).

15. Phillips, D. F., Fleischhauer, A., Mair, A., Walsworth, R. L. \& Lukin, M. D. Storage of light in atomic vapor. Phys. Rev. Lett. 86, 783-786 (2001).

16. Juzeliūnas, G. \& Carmichael, H. J. Systematic formulation of slow polaritons in atomic gases. Phys. Rev. A 65, 021601(R) (2002).

17. Longdell, J. J., Fraval, E., Sellars, M. J. \& Manson, N. B. Stopped light with storage times greater than one second using electromagnetically induced transparency in a solid. Phys. Rev. Lett. 95, 063601 (2005).

18. Schnorrberger, U. et al. Electromagnetically induced transparency and light storage in an atomic Mott insulator. Phys. Rev. Lett. 103, 033003 (2009).

19. Zhang, R., Garner, S. R. \& Hau, L. V. Creation of long-term coherent optical memory via controlled nonlinear interactions in Bose-Einstein condensates. Phys. Rev. Lett. 103, 233602 (2009).

20. Chen, Y.-H. et al. Coherent optical memory with high storage efficiency and large fractional delay. Phys. Rev. Lett. 110, 083601 (2013).

21. Heinze, G., Hubrich, C. \& Hoffman, T. Stopped light and image storage by electromagnetically induced transparency up to the regime of one minute. Phys. Rev. Lett. 111, 033601 (2013)

22. Bajcsy, M., Zibrov, A. S. \& Lukin, M. D. Stationary pulses of light in an atomic medium. Nature 426, 638-641 (2003).

23. Fleischhauer, M., Otterbach, J. \& Unanyan, R. G. Bose-Einstein condensation of stationary-light polaritons. Phys. Rev. Lett. 101, 163601 (2008).

24. Lin, Y. W. et al. Stationary light pulses in cold atomic media and without Bragg gratings. Phys. Rev. Lett. 102, 213601 (2009).

25. Otterbach, J., Ruseckas, J., Unanyan, R. G., Juzeliūnas, G. \& Fleischhauer, M. Effective magnetic fields for stationary light. Phys. Rev. Lett. 104, 033903 (2010).
26. Schmidt, H. \& Imamoglu, A. Giant Kerr nonlinearities obtained by electromagnetically induced transparency. Opt. Lett. 21, 1936-1938 (1996).

27. Harris, S. E. \& Yamamoto, Y. Photon switching by quantum interference. Phys. Rev. Lett. 81, 3611-3614 (1998).

28. Wang, Z. B., Marzlin, K.-P. \& Sanders, B. C. Large cross-phase modulation between slow copropagating weak pulses in ${ }^{87}$ Rb. Phys. Rev. Lett. 97, 063901 (2006).

29. Shiau, B.-W., Wu, M.-C., Lin, C.-C. \& Chen, Y.-C. Low-light-level cross-phase modulation with double slow light pulses. Phys. Rev. Lett. 106, 193006 (2011).

30. Chen, Y.-H. et al. Demonstration of the interaction between two stopped light pulses. Phys. Rev. Lett. 108, 173603 (2012).

31. Venkataraman, V., Saha, K. \& Gaeta, A. L. Phase modulation at the few-photon level for weak-nonlinearity-based quantum computing. Nat. Photon. 7, 138-141 (2013).

32. Chen, W. et al. All-optical switch and transistor gated by one stored photon. Science 341, 768-770 (2013).

33. Peyronel, T. et al. Quantum nonlinear optics with single photons enabled by strongly interacting atoms. Nature 488, 57-60 (2012).

34. Maxwell, D. et al. Storage and control of optical photons using Rydberg polaritons. Phys. Rev. Lett. 110, 103001 (2013).

35. Baur, S., Tiarks, D., Rempe, G. \& Dürr, S. Single-photon switch based on Rydberg blockade. Phys. Rev. Lett. 112, 073901 (2014).

36. Duan, L. M., Lukin, M. D., Cirac, J. I. \& Zoller, P. Long-distance quantum communication with atomic ensembles and linear optics. Nature 414, 413-418 (2001).

37. Balić, V., Braje, D. A., Kolchin, P., Yin, G. Y. \& Harris, S. E. Generation of paired photons with controllable waveforms. Phys. Rev. Lett. 94, 183601 (2005).

38. Chanelière, T. et al. Storage and retrieval of single photons transmitted between remote quantum memories. Nature 438, 833-836 (2005).

39. Eisaman, M. D. et al. Electromagnetically induced transparency with tunable single-photon pulses. Nature 438, 837-841 (2005).

40. Choi, K. S., Deng, H., Laurat, J. \& Kimble, H. J. Mapping photonic entanglement into and out of a quantum memory. Nature 452, 67-71 (2008)

41. Zhao, B. et al. A millisecond quantum memory for scalable quantum networks. Nat. Phys. 5, $95-99$ (2009).

42. Zhou, S. et al. Optimal storage and retrieval of single-photon waveforms. Opt. Express 20, 24124-24131 (2012).

43. Harris, S. E. Electromagnetically induced transparency. Phys. Today 50, 36-42 (1997).

44. Lukin, M. D. Colloquium: trapping and manipulating photon states in atomic ensembles. Rev. Mod. Phys. 75, 457-472 (2003).

45. Fleischhauer, M., Imamoglu, A. \& Marangos, J. P. Electromagnetically induced transparency: optics in coherent media. Rev. Mod. Phys. 77, 633-673 (2005).

46. Unanyan, R. G. et al. Spinor slow-light and dirac particles with variable mass. Phys. Rev. Lett. 105, 173603 (2010).

47. Ruseckas, J. et al. Photonic-band-gap properties for two-component slow light. Phys. Rev. A 83, 063811 (2011).

48. Ruseckas, J., Kudriašov, V., Yu, I. A. \& Juzeliūnas, G. Transfer of orbital angular momentum of light using two-component slow light. Phys. Rev. A 87, 053840 (2013).

49. Bao, Q.-Q. et al. Coherent generation and dynamic manipulation of double stationary light pulses in a five-level double-tripod system of cold atoms. Phys Rev. A 84, 063812 (2011).

50. Petrosyan, D. \& Malakyan, Y. P. Magneto-optical rotation and cross-phase modulation via coherently driven four-level atoms in a tripod configuration. Phys. Rev. A 70, 023822 (2004).

51. Raczyński, A., Zaremba, J. \& Zielińska-Kaniasty, S. Beam splitting and HongOu-Mandel interference for stored light. Phys. Rev. A 75, 013810 (2007).

52. Wang, H.-H. et al. Slowing and storage of double light pulses in a $\operatorname{Pr}^{3+}: \mathrm{Y}_{2} \mathrm{SiO}_{5}$ crystal. Opt. Lett. 34, 2596-2598 (2009).

53. Karpa, L., Vewinger, F. \& Weitz, M. Resonance beating of light stored using atomic spinor polaritons. Phys. Rev. Lett. 101, 170406 (2008).

54. Ruseckas, J., Mekys, A. \& Juzeliūnas, G. Slow polaritons with orbital angular momentum in atomic gases. Phys. Rev. A 83, 023812 (2011).

55. Chiu, C.-K. et al. Low-light-level four-wave mixing by quantum interference. Phys. Rev. A 89, 023839 (2014)

56. Braje, D. A., Balić, V., Goda, S., Yin, G. Y. \& Harris, S. E. Frequency mixing using electromagnetically induced transparency in cold atoms. Phys. Rev. Lett. 93, 183601 (2004).

57. Lin, Y.-W., Chou, H.-C., Dwivedi, P. P., Chen, Y.-C. \& Yu, I. A. Using a pair of rectangular coils in the MOT for the production of cold atom clouds with large optical density. Opt. Express 16, 3753-3761 (2008).

\section{Acknowledgements}

This work was supported by the Ministry of Science and Technology of Taiwan under Grant Nos. 101-2112-M-007-008-MY3, 103-2923-M-007-001 and 103-2119-M-007-011, by the project TAP LLT $01 / 2012$ of the Research Council of Lithuania and the Ministry of Science and Technology of Taiwan, and by the EU FP7 IRSES project COLIMA (contract PIRSES-GA-2009-247475). 


\section{Author contributions}

I.A.Y., G.J., J.R., V.K. and H.-W.C. conceived the experiment. I.A.Y. and M.-J.L. designed the experimental setup and methods. M.-J.L., C.-Y.L., K.-F.C. and H.-W.C. built the setup and carried out the experiment. M.-J.L. and C.-Y.L. calculated the predictions and analysed the data under the supervision of I.A.Y. The manuscript was written by I.A.Y. with help from G.J., M.-J.L., J.R., C.-Y.L. and V.K.

\section{Additional information}

Supplementary Information accompanies this paper at http://www.nature.com/ naturecommunications

Competing financial interests: The authors declare no competing financial interests.
Reprints and permission information is available online at http://npg.nature.com/ reprintsandpermissions/

How to cite this article: Lee, M.-J. L. et al. Experimental demonstration of spinor slow light. Nat. Commun. 5:5542 doi: 10.1038/ncomms6542 (2014).

\section{(c) (1)}

This work is licensed under a Creative Commons Attribution 4.0 International License. The images or other third party material in this article are included in the article's Creative Commons license, unless indicated otherwise in the credit line; if the material is not included under the Creative Commons license, users will need to obtain permission from the license holder to reproduce the material. To view a copy of this license, visit http://creativecommons.org/licenses/by/4.0/ 